\title{
Strong bounds on perturbations
}

\author{
Bernd Heidergott • Arie Hordijk • Haralambie Leahu
}

Received: 12 November 2007 / Accepted: 21 May 2008 / Published online: 1 July 2008

(C) The Author(s) 2008

\begin{abstract}
This paper provides strong bounds on perturbations over a collection of independent random variables, where 'strong' has to be understood as uniform w.r.t. some functional norm. Our analysis is based on studying the concept of weak differentiability. By applying a fundamental result from the theory of Banach spaces, we show that weak differentiability implies norm Lipschitz continuity. This result leads to bounds on the sensitivity of finite products of probability measures, in norm sense. We apply our results to derive bounds on perturbations for the transient waiting times in a $G / G / 1$ queue.
\end{abstract}

Keywords Weak differentiation · Banach space $\cdot$ Strong convergence · Perturbation analysis

\section{Introduction}

A wide range of probabilistic models in the area of manufacturing, transportation, finance and communication can be modeled by studying cost functions over a finite

This research is supported by the Technology Foundation STW, applied science division of NWO and the technology programme of the Ministry of Economic Affairs.

B. Heidergott $(\varangle) \cdot$ H. Leahu

Vrije Universiteit, Amsterdam, The Netherlands

e-mail: bheidergott@feweb.vu.nl

H. Leahu

e-mail: hleahu@feweb.vu.nl

A. Hordijk

Leiden Universiteit, Leiden, The Netherlands

e-mail: arie-hordijk@xs4all.nl 
collection of random variables. More specifically, letting $\mu_{i, \theta}$ be a probability measure on some state space $\mathbb{S}_{i}$ (for $1 \leq i \leq n$ ) depending on some parameter $\theta$, then this paper is concerned with the following type of models

$J_{g}(\theta):=\mathbb{E}_{\theta}\left[g\left(X_{n}, \ldots, X_{1}\right)\right]=\int \cdots \int g\left(s_{n}, \ldots, s_{1}\right) \mu_{n, \theta}\left(d s_{n}\right) \cdots \mu_{1, \theta}\left(d s_{1}\right)$,

for a real-valued cost function $g$, where $X_{i}$ is distributed according to $\mu_{i, \theta}$; and we assume, for ease of exposition, that $\theta \in \Theta=(a, b) \subset \mathbb{R}$, with $a<b$, as the extension to vector-valued parameters with $\Theta \subset \mathbb{R}^{n}$ is straightforward. This class of models contains, for example, transient waiting times in queueing networks or insurance models over a finite number of claims. If $\theta_{1}, \theta_{2} \in \Theta$ then this paper studies the following type of bounds:

(i) Bounds on $\left|J_{g}\left(\theta_{2}\right)-J_{g}\left(\theta_{1}\right)\right|$, for some specified cost function $g$.

(ii) Uniform bounds with respect to a class $\mathcal{D}$ of cost functions, i.e., for

$$
\sup _{g \in \mathcal{D}}\left|J_{g}\left(\theta_{2}\right)-J_{g}\left(\theta_{1}\right)\right|
$$

Starting point of the analysis put forward in this paper is that weak differentiability of the probability measures $\mu_{i, \theta}, 1 \leq i \leq n$, in (1) (to be defined below) implies local Lipschitz continuity of $J_{g}(\theta)$ as a function of $\theta$, which leads to type (i) bounds. For type (ii) bounds, we will work with a norm framework where we will use the weighted supremum norm or $v$-norm (to be defined below). Elaborating on a link between functional analysis and weak differentiability, we will show that $J_{g}(\theta)$ is $v$-norm Lipschitz, where the norm-Lipschitz constant can be obtained as a simple function of the norm-Lipschitz constant of the individual probability measures $\mu_{i, \theta}$. Moreover, the Lipschitz constant of a probability measure can be computed in a simple way from the weak derivative of the probability measure. Classical theory on $v$-norms assumes that the mapping $v$ is bounded away from zero, i.e, $\inf _{s} v(s)>0$. As it will turn out, this is not efficient when one establishes type (ii) bounds, and we will show how the definition of $v$-norm extends to the case where $v$ may have zeros.

Norm bounds for Markov chains of the type developed in this paper have been intensively studied in the literature on perturbation analysis of Markov chains, which goes back to the pioneering paper of Schweitzer (1968). In this line of research one tries to bound the distance between the stationary distributions of two Markov chains by the difference between the corresponding Markov kernels, where the "distance" is measured by an appropriate norm. See Cho and Meyer (2001) for an overview on these results. The use of the weighted supremum norm for establishing norm bounds goes back to Lipman (1974) and the weighted supremum norm has been frequently used in Markov decision theory (Dekker and Hordijk 1988; Hordijk and Yushkevich 1999) and stability theory of Markov chains (Meyn and Tweedie 1993). A key element of the approach presented in this paper is that we assume that the function $v$ is of exponential type, i.e., $v(s)=e^{\alpha s}$ for $s \geq 0$ and some $\alpha>0$, which allows for a certain flexibility in choosing a convenient value for $\alpha$. This approach is inspired by strong stable Markov chains (Aissani and Bouallouche-Medjkoune 2006; Kartashov 
1996), where this technique is used to identify the stability neighborhood of a Markov chain. The paper deviates from the above approaches as it studies finite products of distributions, in contrast to stationary distributions of Markov chains, and in that it investigates to what extent a normed based bounding approach is of practical use in terms of actually computing bounds numerically.

The paper is organized as follows. In Sect. 1, we present basic notions for studying the limiting behavior of sequences of measures and weak differentiability is introduced. In addition, we show how can one establish bounds on perturbations using weak derivatives. In Sect. 2, we concentrate on perturbations of the waiting times in the $G / G / 1$ queue and we establish bounds on perturbations for transient waiting times. The contributions of the paper are summarized in Sect. 3.

\section{Weak differentiation of probability measures}

In this section, we present an efficient method for deriving uniform bounds on perturbations for parameter-dependent stochastic systems. This is possible by means of weak derivatives. To this end, we start with an overview of the basic concepts in Sect. 1.1 and we present a rule for weak differentiation of product measures in Sect. 1.2. In Sect. 1.3, we properly define the concept of strong convergence, by means of the $v$-norm and establish a relation between weak differentiability and strong Lipschitz continuity that is essential for our analysis. Eventually, bounds on perturbations for some usual distributions are established in Sect. 1.4 and we present an application in Sect. 1.5.

\subsection{Weak properties of measure-valued mappings}

This section provides a short introduction to the theory of measure-valued differentiation. For the ease of exposure we list a few notations that we will use throughout this paper. Let $(\mathbb{S}, d)$ be a separable ${ }^{1}$ metric space endowed with the Borel field $\mathcal{S}$ :

- $\mathcal{C}(\mathbb{S})$ denotes the set of real-valued continuous mappings on $\mathbb{S}$.

- $\mathcal{C}_{B}(\mathbb{S}) \subset \mathcal{C}(\mathbb{S})$ denotes the subset of bounded mappings.

- $\mathcal{C}^{+}(\mathbb{S}) \subset \mathcal{C}(\mathbb{S})$ denotes the subset of non-negative mappings.

- $\mathcal{M}(\mathcal{S})$ denotes the space of finite, regular (signed) measures on $(\mathbb{S}, \mathcal{S})$.

- $\mathcal{M}^{+}(\mathcal{S}) \subset \mathcal{M}(\mathcal{S})$ denotes the cone of positive measures, i.e.,

$$
\mathcal{M}^{+}(\mathcal{S}):=\{\mu \in \mathcal{M}(\mathcal{S}): \mu(E) \geq 0, \forall E \in \mathcal{S}\} .
$$

- $\mathcal{M}^{1}(\mathcal{S}) \subset \mathcal{M}^{+}(\mathcal{S})$ denotes the set of probability measures, i.e., $\mu(\mathbb{S})=1$.

- for $\mu \in \mathcal{M}$ we denote by $\mathcal{L}^{1}(\mu)$ the set of measurable mappings which are integrable w.r.t. $\mu$ and, for a family $\left\{\mu_{i}: i \in I\right\} \subset \mathcal{M}(\mathcal{S})$, let

$$
\mathcal{L}^{1}\left(\mu_{i}: i \in I\right):=\bigcap_{i \in I} \mathcal{L}^{1}\left(\mu_{i}\right)
$$

\footnotetext{
1 A metric space $(\mathbb{S}, d)$ is called separable if it contains a countable dense subset.
} 
In the following, we omit specifying the underlying space $\mathbb{S}$ (resp., Borel field $\mathcal{S}$ ) when no confusion occurs.

Let $\left\{\mu_{n}\right\}_{n}$ be a sequence of measures in $\mathcal{M}$. For $\mathcal{D} \subset \mathcal{L}^{1}\left(\mu_{n}: n \in \mathbb{N}\right)$, we call $\mu_{n}$ weakly $\mathcal{D}$-convergent if there exists $\mu \in \mathcal{M}$ such that

$$
\forall g \in \mathcal{D}: \quad \lim _{n \rightarrow \infty} \int g(s) \mu_{n}(d s)=\int g(s) \mu(d s) .
$$

We call $\mu$ in (2) a weak $\mathcal{D}$-limit of $\left\{\mu_{n}\right\}_{n}$ and we note that, in general, the limit in (2) is not unique. Moreover, we can assume w.l.o.g. that $\mathcal{D}$ is a linear space, since the property in (2) extends in a straightforward way to the linear space generated by $\mathcal{D}$. Taking $\mathcal{D}=\mathcal{C}_{B}$ in (2), yields classical weak convergence of measures which defines the concept of convergence in distribution. Moreover, in his pioneering work on weak derivatives, Pflug used $\mathcal{D}=\mathcal{C}_{B}$ for defining weak derivatives; see Pflug (1996). The main reason behind this choice is that for $\mathcal{D}=\mathcal{C}_{B}$ the weak limit in (2) is uniquely determined.

Definition 1 For $v \in \mathcal{C}^{+}$, let $\mathcal{C}_{v}$ denote the set of continuous mappings that are bounded by a multiple of $v$; in formula:

$$
\mathcal{C}_{v}:=\{g \in \mathcal{C}|\exists c \geq 0:| g(s) \mid \leq c \cdot v(s), \forall s \in \mathbb{S}\}
$$

We call $\mathcal{C}_{v}$ the space of continuous $v$-bounded mappings on $S$.

Note that, for $\mu \in \mathcal{M}$, we have $\mathcal{C}_{v} \subset \mathcal{L}^{1}(\mu)$ if and only if $v \in \mathcal{L}^{1}(\mu)$. It is straightforward that $\mathcal{C}_{v}$ is a linear subspace of $\mathcal{C}$ and for $v \equiv 1$ we have $\mathcal{C}_{v}=\mathcal{C}_{B}$. Moreover, as shown by Lemma 3 in the Appendix, the weak $\mathcal{C}_{v}$-limit is uniquely determined up to an equivalence relation. Hence, the condition $\inf _{s \in \mathbb{S}} v(s)>0$, which implies $\mathcal{C}_{B} \subset \mathcal{C}_{v}$ and consequently the uniqueness of the $\mathcal{C}_{v}$-limit is not crucial for our analysis.

Example 1 Let $v \in \mathcal{C}^{+}([0, \infty))$ be defined as $v(x)=e^{\alpha x}$, for some $\alpha>0$. Since for every polynomial $P$ it holds that

$$
\lim _{x \rightarrow \infty} e^{-\alpha x} P(x)=0
$$

it turns out that the space $\mathcal{C}_{v}([0, \infty))$ contains all (finite) polynomials. However, the polynomials are not the only elements of $\mathcal{C}_{v}$ since, for instance, the mapping $x \mapsto$ $\ln (1+x)$ also belongs to $\mathcal{C}_{v}$.

The next result relates general $\mathcal{C}_{v}$-convergence to classical $\mathcal{C}_{B}$-convergence.

Lemma 1 Let $\left(\mu_{n}: n \in \mathbb{N}\right) \subset \mathcal{M}^{1}$ and $v \in \mathcal{C}^{+}$be s.t. $v \in \mathcal{L}^{1}\left(\mu_{n}: n \in \mathbb{N}\right)$ and $\mu_{n} \stackrel{\mathcal{C}_{B}}{\Longrightarrow} \mu$, for some $\mu \in \mathcal{M}^{1}$, i.e., $\mu$ is the classical weak limit of the sequence $\left\{\mu_{n}\right\}_{n}$. Assume further that

$$
\lim _{n \rightarrow \infty} \int v(x) \mu_{n}(d x)=\int v(x) \mu(d x) .
$$


Then, $\mu_{n} \stackrel{\mathcal{C}_{v}}{\Longrightarrow} \mu$, i.e., $\mu$ is also the weak $\mathcal{C}_{v}$-limit of the sequence $\left\{\mu_{n}\right\}_{n}$.

Proof One has to show that the limit relation in (2) holds true for $\mathcal{D}=\mathcal{C}_{v}$. By hypothesis, the conclusion is equivalent to uniform integrability of each $g \in \mathcal{C}_{v}$ w.r.t. the family $\left\{\mu_{n}: n \in \mathbb{N}\right\}$; see, e.g., Billingsley (1966). That is

$$
\forall g \in \mathcal{C}_{v}: \lim _{\alpha \rightarrow \infty} \sup _{n \in \mathbb{N}} \int|g(s)| \cdot \mathbb{I}_{\{|g|>\alpha\}}(s) \mu_{n}(d s)=0,
$$

where $\mathbb{I}_{\{|g|>\alpha\}}(s)$ denotes the indicator function of the set $\{s:|g(s)|>\alpha\}$. One can easily show that a sufficient condition for (5) is the uniform integrability of $v$ w.r.t. the family $\left\{\mu_{n}: n \in \mathbb{N}\right\}$ which follows from (4).

Remark 1 Lemma 1 essentially says that uniform integrability of $v$, w.r.t. the sequence $\left\{\mu_{n}\right\}_{n}$ is a sufficient condition for $\mathcal{C}_{v}$-convergence, provided that $\mu_{n}$ converges in classical sense.

As illustrated by Lemma $1, \mathcal{C}_{v}$-spaces are suitable choices for defining weak convergence because of their structure. In particular, applying Lemma 1 to $\mathcal{C}_{v}$ defined in Example 1, we conclude that if the sequence $\left\{\mu_{n}\right\}_{n}$ converges weakly to $\mu$ (in the classical sense) and (2) holds true for $v(x)=e^{\alpha x}$ then the moments of $\mu_{n}$ converge to those of $\mu$.

Consider a family $\left\{\mu_{\theta}: \theta \in \Theta\right\} \subset \mathcal{M}$, with $\Theta:=(a, b) \subset \mathbb{R}$, for some $a<b$. The correspondence $\theta \mapsto \mu_{\theta}$ defines a measure-valued mapping from $\Theta$ to $\mathcal{M}$. For a convenient notation, we agree to identify this mapping with its values, i.e., we use the notation $\mu_{\theta}$ to illustrate the functional dependence w.r.t. $\theta$, rather than specifying a particular value.

Definition 2 Let $\left\{\mu_{\theta}: \theta \in \Theta\right\} \subset \mathcal{M}^{1}$ and $v \in \mathcal{C}^{+} \cap \mathcal{L}^{1}\left(\mu_{\theta}: \theta \in \Theta\right)$. We say that $\mu_{\theta}$ is weakly $\mathcal{C}_{v}$-continuous if the measure-valued mapping $\theta \mapsto \mu_{\theta}$ is weakly $\mathcal{C}_{v}$-continuous at $\theta$, i.e.,

$$
\forall g \in \mathcal{C}_{v}: \lim _{\xi \rightarrow 0} \int g(s) \mu_{\theta+\xi}(d s)=\int g(s) \mu_{\theta}(d s)
$$

We say that $\mu_{\theta}$ is weakly $\mathcal{C}_{v}$-continuous on $\Theta$ if, for each $\theta \in \Theta, \mu_{\theta}$ is weakly $\mathcal{C}_{v}$-continuous, i.e., the measure-valued mapping $\theta \mapsto \mu_{\theta}$ is weakly $\mathcal{C}_{v}$-continuous at $\theta$, for all $\theta \in \Theta$.

In the same vein, we say that $\mu_{\theta}$ is weakly $\mathcal{C}_{v}$-differentiable if there exists $\mu_{\theta}^{\prime} \in \mathcal{M}$ such that

$$
\forall g \in \mathcal{C}_{v}: \lim _{\xi \rightarrow 0} \frac{1}{\xi}\left(\int g(s) \mu_{\theta+\xi}(d s)-\int g(s) \mu_{\theta}(d s)\right)=\int g(s) \mu_{\theta}^{\prime}(d s)
$$

and we say that $\mu_{\theta}$ is weakly $\mathcal{C}_{v}$-differentiable on $\Theta$ if, for each $\theta \in \Theta, \mu_{\theta}$ is weakly $\mathcal{C}_{v}$-differentiable. 
In the following, we omit specifying the set $\mathcal{C}_{v}$ when no confusion occurs and we use expressions such as "weakly convergent", "weak limit", "weakly continuous" and "weakly differentiable" instead.

Note that, if $\mu_{\theta}$ is weakly differentiable, then $\mu_{\theta}^{\prime}$ is a finite signed measure. Any finite signed measure $\mu$ can be written in a unique way as the difference between two orthogonal $^{2}$ finite positive measures, denoted by $[\mu]^{+}$and $[\mu]^{-}$. The fact is known as the Hahn-Jordan decomposition. Hence, it holds for $\mu_{\theta}^{\prime}$ that:

$$
\forall E \in \mathcal{S}: \mu_{\theta}^{\prime}(E)=\left[\mu_{\theta}^{\prime}\right]^{+}(E)-\left[\mu_{\theta}^{\prime}\right]^{-}(E)
$$

Since $\mu_{\theta+\xi}(\mathbb{S})=\mu_{\theta}(\mathbb{S})=1$, for all $\xi$, and since $\mu_{\theta}^{\prime}$ is the weak limit of the family $\left\{\left(\mu_{\theta+\xi}-\mu_{\theta}\right) / \xi\right\}_{\xi}$ as $\xi \rightarrow 0$, it follows that $\mu_{\theta}^{\prime}(\mathbb{S})=0$; see the Appendix for details. This justifies the following definition:

$$
\forall \theta \in \Theta:\left[\mu_{\theta}^{\prime}\right]^{+}(\mathbb{S})=\left[\mu_{\theta}^{\prime}\right]^{-}(\mathbb{S}):=c_{\theta}
$$

This yields the following representation of $\mu_{\theta}^{\prime}$ :

$$
\forall E \in \mathcal{S}: \mu_{\theta}^{\prime}(E)=c_{\theta} \mu_{\theta}^{+}(E)-c_{\theta} \mu_{\theta}^{-}(E)
$$

where $\mu_{\theta}^{+}=c_{\theta}^{-1}\left[\mu_{\theta}^{\prime}\right]^{+} \in \mathcal{M}^{1}(\mathcal{S})$ and $\mu_{\theta}^{-}=c_{\theta}^{-1}\left[\mu_{\theta}^{\prime}\right]^{-} \in \mathcal{M}^{1}(\mathcal{S})$.

The variation of $\mu_{\theta}^{\prime}$, with decomposition as in (7), is defined by

$$
\forall E \in \mathcal{S}:\left|\mu_{\theta}^{\prime}\right|(E):=c_{\theta} \mu_{\theta}^{+}(E)+c_{\theta} \mu_{\theta}^{-}(E) .
$$

Letting $E=\mathbb{S}$ in the above definition, we obtain the total variation of $\mu_{\theta}^{\prime}$, i.e.:

$$
\left\|\mu_{\theta}^{\prime}\right\|:=\left|\mu_{\theta}^{\prime}\right|(\mathbb{S})=c_{\theta} \mu_{\theta}^{+}(\mathbb{S})+c_{\theta} \mu_{\theta}^{-}(\mathbb{S})
$$

Note that the decomposition in (6) is by means of positive measures, whereas the decomposition in (7) is by means of probability measures. In accordance with (7), $\mu_{\theta}^{\prime}$ is completely characterized through the triple $\left(c_{\theta}, \mu_{\theta}^{+}, \mu_{\theta}^{-}\right)$, with $\mu_{\theta}^{+}, \mu_{\theta}^{-} \in \mathcal{M}^{1}(\mathcal{S})$. This representation has a clear advantage over (6) as it can be interpreted in terms of random variables and thus can be made fruitful for simulation. More specifically, let $\mu_{\theta}$ be weakly differentiable with weak derivative $\left(c_{\theta}, \mu_{\theta}^{+}, \mu_{\theta}^{-}\right)$satisfying (7). Then for any $g \in \mathcal{C}_{v}$ it holds that

$$
\frac{d}{d \theta} \mathbb{E}\left[g\left(X_{\theta}\right)\right]=c_{\theta} \mathbb{E}\left[g\left(X_{\theta}^{+}\right)-g\left(X_{\theta}^{-}\right)\right]
$$

where $X_{\theta}, X_{\theta}^{+}, X_{\theta}^{-}$have distributions $\mu_{\theta}, \mu_{\theta}^{+}, \mu_{\theta}^{-}$, respectively. This leads to the following definition.

2 Two measures $\mu, \eta$ defined on a common measurable space $(\mathbb{S}, \mathcal{S})$ are said to be orthogonal if there exists a set $A \in \mathcal{S}$ such that $\mu(\mathbb{S} \backslash A)=\eta(A)=0$. That is to say that the two measures, $\mu$ and $\eta$ have disjoint support. 
Definition 3 Let $\mu_{\theta} \in \mathcal{M}^{1}$ be weakly differentiable. We call a triple $\left(c_{\theta}, \mu_{\theta}^{+}, \mu_{\theta}^{-}\right)$, with $\mu_{\theta}^{ \pm} \in \mathcal{M}^{1}$ and $c_{\theta} \in \mathbb{R}$, a weak derivative of $\mu_{\theta}$ if it holds that:

$$
\forall g \in \mathcal{C}_{v}: \int g(s) \mu_{\theta}^{\prime}(d s)=c_{\theta}\left(\int g(s) \mu_{\theta}^{+}(d s)-\int g(s) \mu_{\theta}^{-}(d s)\right) .
$$

A weak $\mathcal{C}_{v}$-derivative, cf. Definition 3 , is not unique and an instance of the derivative can always be found through the Hahn-Jordan decomposition. The weak derivative of a probability measure $\mu_{\theta}$ becomes unique if we require that $\mu_{\theta}^{+}$and $\mu_{\theta}^{-}$are orthogonal, in which case $c_{\theta}$ is minimized. However, in applications it is not always the case that the orthogonal representation is the most convenient one. This fact is illustrated by the following example.

Example 2 Let $\mathbb{S}=[0, \infty), v(s)=s^{p}$, for some $p \geq 1$ and let

$$
\forall s>0: f_{\theta}(s):=\theta e^{-\theta s},
$$

be the density of the exponential distribution with rate $\theta$. Take $\Theta=(a, b)$, for $0<$ $a<b<\infty$, and $\mu_{\theta}(d s)=f_{\theta}(s) \cdot d s$, for $\theta \in \Theta$. Then, $\mu_{\theta}$ is $\mathcal{C}_{v}$-differentiable, which can be easily seen by applying the Dominated Convergence Theorem. Indeed, one can directly differentiate the density $f_{\theta}(s)$ w.r.t. $\theta$, which yields

$$
\frac{d}{d \theta} f_{\theta}(s)=(1-\theta s) e^{-\theta s}
$$

Letting $c_{\theta}=(\theta e)^{-1}$ and introducing densities

$$
f_{\theta}^{+}(s):=\frac{1}{c_{\theta}} \mathbb{I}_{[0,1 / \theta]}(s)(1-\theta s) e^{-\theta s}, \quad \forall s>0,
$$

and

$$
f_{\theta}^{-}(s):=\frac{1}{c_{\theta}} \mathbb{I}_{[1 / \theta, \infty)}(s)(\theta s-1) e^{-\theta s}, \quad \forall s>0,
$$

we have $\frac{d}{d \theta} f_{\theta}(s)=c_{\theta} f_{\theta}^{+}(d s)-c_{\theta} f_{\theta}^{-}(d s)$ and we obtain:

$$
\forall g \in \mathcal{C}_{v}: \frac{d}{d \theta} \int g(s) \mu_{\theta}(d s)=c_{\theta}\left(\int g(s) f_{\theta}^{+}(s) d s-\int g(s) f_{\theta}^{-}(s) d s\right)
$$

and a weak derivative of $\mu_{\theta}$ is given by

$$
\mu_{\theta}^{\prime}(d s)=\left(c_{\theta}, f_{\theta}^{+}(s) d s, f_{\theta}^{-}(s) d s\right)
$$

Note that this is the orthogonal representation. However, one can also write

$$
\frac{d}{d \theta} f_{\theta}(s)=\frac{1}{\theta}\left(\theta e^{-\theta s}-\theta^{2} s e^{-\theta s}\right),
$$


which yields another representation for the weak derivative. That is

$$
\mu_{\theta}^{\prime}(d s)=\left(\frac{1}{\theta}, \mu_{\theta}(d s), \gamma_{2, \theta}(d s)\right)
$$

where $\gamma_{2, \theta}$ denotes the convolution of two exponential distributions with rate $\theta$. Obviously, this representation is more meaningful in terms of random variables.

In the above example, $\mu_{\theta}$ as well as $\mu_{\theta}^{+}$and $\mu_{\theta}^{-}$have Lebesgue densities, that is, $\mu_{\theta}$ as well as its weak derivative $\mu_{\theta}^{\prime}$ are absolutely continuous w.r.t. the same measure. The following example shows that this is not always the case.

Example 3 Let $v \in \mathcal{C}^{+}([0, \infty])$ and $\psi_{\theta}$ denote the uniform distribution on the interval $[0, \theta]$. Then it holds that

$$
\begin{aligned}
\forall g \in \mathcal{C}_{v}: \frac{d}{d \theta} \int g(x) \psi_{\theta}(d x) & =\frac{d}{d \theta}\left(\frac{1}{\theta} \int_{0}^{\theta} g(x) d x\right)=\frac{1}{\theta} g(\theta)-\frac{1}{\theta^{2}} \int_{0}^{\theta} g(x) d x \\
& =\frac{1}{\theta}\left(\int g(x) \delta_{\theta}(d x)-\int g(x) \psi_{\theta}(d x)\right),
\end{aligned}
$$

where $\delta_{x}$ denotes the Dirac measure in $x$. Hence, $\left(1 / \theta, \delta_{\theta}, \psi_{\theta}\right)$ is a $\mathcal{C}_{v}$-derivative of $\psi_{\theta}$. Note that the $\mathcal{C}_{v}$-derivative of $\psi_{\theta}$ has a continuous and a discrete part while $\psi_{\theta}$ is a continuous distribution, for a detailed discussion see Heidergott and Hordijk (2004).

Remark 2 In the literature, differentiation of a probability measure $\mu_{\theta}$ has also been defined as differentiability of the corresponding set function, that is:

$$
\mu_{\theta}^{\prime}(A)=\frac{d}{d \theta} \mu_{\theta}(A), \quad \forall A \in \mathcal{S}
$$

see, e.g., Kushner and Vázquez-Abad (1992). This definition can be recovered by extending $\mathcal{C}_{v}$ with the set of indicator functions. However, this is an unfortunate choice. Roughly speaking, there are too few probability measures that are differentiable according to this definition. The following example illustrates this. Taking $\psi_{\theta}$ to be the uniform distribution on $[0, \theta]$ and denoting the Lebesgue measure by $\lambda$ it holds

$$
\forall x>0: \psi_{\theta}([0, x])=\frac{1}{\theta} \lambda([0, x] \cap[0, \theta])=\frac{1}{\theta} \min (x, \theta) .
$$

At $\theta=x$, the left-hand side derivative of $\psi_{\theta}([0, x])$ equals 0 whereas the right-hand side derivative equals $-1 / x$. Hence, $\psi_{\theta}$ fails to be weakly differentiable in the set-wise sense, whereas it is shown in Example 3 that $\psi_{\theta}$ is $\mathcal{C}_{v}$-differentiable. 
1.2 Weak differentiation of product measures

Let $\left\{\mu_{i, \theta}: \theta \in \Theta\right\} \subset \mathcal{M}^{1}\left(\mathbb{S}_{i}\right)$, for $1 \leq i \leq n$, be a finite family of measure-valued mappings and let us consider the product mapping given by

$$
\forall \theta \in \Theta: \Pi_{\theta}:=\mu_{1, \theta} \times \cdots \times \mu_{n, \theta} .
$$

If $v_{i} \in \mathcal{C}^{+}\left(\mathbb{S}_{i}\right)$, for $1 \leq i \leq n$, we define $V \in \mathcal{C}^{+}\left(\mathbb{S}_{1} \times \cdots \times \mathbb{S}_{n}\right)$ as follows:

$$
\forall\left(s_{1}, \ldots, s_{n}\right) \in \mathbb{S}_{1} \times \cdots \times \mathbb{S}_{n}: V\left(s_{1}, \ldots, s_{n}\right):=\prod_{i=1}^{n} v_{i}\left(s_{i}\right) .
$$

Then, the following assertion holds true:

Theorem 1 If $\mu_{i, \theta}$ is weakly $\mathcal{C}_{v_{i}}$-differentiable, for $1 \leq i \leq n$, then the product measure $\Pi_{\theta}$ is weakly $\mathcal{C}_{V}$-differentiable. Moreover, if $\left(c_{i, \theta}, \mu_{i, \theta}^{+}, \mu_{i, \theta}^{-}\right)$is a weak derivative of $\mu_{i, \theta}$, for $1 \leq i \leq n$, then an instance of the weak derivative $\Pi_{\theta}^{\prime}$ is given by $\left(C_{\theta}, \Pi_{\theta}^{+}, \Pi_{\theta}^{-}\right)$, where

$$
\begin{aligned}
C_{\theta} & =\sum_{i=1}^{n} c_{i, \theta}, \\
\Pi_{\theta}^{+} & =\sum_{i=1}^{n} \frac{c_{i, \theta}}{C_{\theta}} \cdot \mu_{1, \theta} \times \cdots \times \mu_{i, \theta}^{+} \times \cdots \times \mu_{n, \theta}, \\
\Pi_{\theta}^{-} & =\sum_{i=1}^{n} \frac{c_{i, \theta}}{C_{\theta}} \cdot \mu_{1, \theta} \times \cdots \times \mu_{i, \theta}^{-} \times \cdots \times \mu_{n, \theta} .
\end{aligned}
$$

In particular, if $\mu_{1, \theta}=\cdots=\mu_{n, \theta}=\mu_{\theta}$ and $\mu_{\theta}^{\prime}=\left(c_{\theta}, \mu_{\theta}^{+}, \mu_{\theta}^{-}\right)$it holds that

$$
\left(\mu_{\theta}^{n}\right)^{\prime}=\left(n c_{\theta}, \frac{1}{n} \sum_{i=1}^{n} \mu_{\theta}^{i-1} \times \mu_{\theta}^{+} \times \mu_{\theta}^{n-i}, \frac{1}{n} \sum_{i=1}^{n} \mu_{\theta}^{i-1} \times \mu_{\theta}^{-} \times \mu_{\theta}^{n-i}\right),
$$

where, for $k \geq 1, \mu_{\theta}^{k}$ denotes the $k$-fold product of $\mu_{\theta}$ and we ignore the term $\mu_{\theta}^{0}$.

For more details on rules of weak differentiation of product measures, including the proof of Theorem 1, we refer to Heidergott and Leahu (2008).

Example 4 Let us consider a $G / G / 1$ queue where the service times have distribution $\mu$ and inter-arrival times have distribution $\eta$. If $\left\{W_{n}: n \geq 1\right\}$ denotes the sequence of waiting times, then Lindley's recursion yields:

$$
\forall n \geq 1: W_{n+1}=\max \left\{W_{n}+S_{n}-A_{n+1}, 0\right\},
$$


where $\left\{S_{n}: n \geq 1\right\}$ and $\left\{A_{n}: n \geq 1\right\}$ denote the sequence of service and inter-arrival times, respectively. To apply Theorem 1 let us consider the sequence of mappings $\left\{g_{n}: n \geq 1\right\}, g_{n} \in \mathcal{C}\left(\mathbb{R}^{2 n}\right)$ defined as $g_{1}(s, t)=\max \{s-t, 0\}$ and

$$
\forall n \geq 1, \sigma, \tau \in \mathbb{R}^{n}, s, t \in \mathbb{R}: g_{n+1}(\sigma, s, \tau, t)=\max \left\{g_{n}(\sigma, \tau)+s-t, 0\right\} .
$$

It follows from Lindley's recursion that the waiting times satisfy

$$
\forall n \geq 1: W_{n+1}=g_{n}\left(S_{1}, \ldots, S_{n}, A_{2}, \ldots, A_{n+1}\right)
$$

Hence, if the service times $S_{1}, \ldots, S_{n}, \ldots$ have distribution $\mu_{\theta}$, inter-arrival times have distribution $\eta$, independent of $\theta$, and $\mu_{\theta}$ is $\mathcal{C}_{v}$-differentiable then Theorem 1 implies that for all $\bar{v} \in \mathcal{C}^{+}([0, \infty))$ satisfying

$$
\sup _{\substack{s_{1}, \ldots, s_{n} \in \mathbb{R} \\ t_{1}, \ldots, t_{n} \in \mathbb{R}}} \frac{\bar{v}\left(g_{n}\left(s_{1}, \ldots, s_{n}, t_{1}, \ldots, t_{n}\right)\right)}{\prod_{i=1}^{n} v\left(s_{i}\right) \prod_{i=1}^{n} v\left(t_{i}\right)}<\infty
$$

the distribution of $W_{n+1}$ is weakly $\mathcal{C}_{\bar{v}}$-differentiable and it holds that

$$
\forall f \in \mathcal{C}_{\bar{v}}: \frac{d}{d \theta} \mathbb{E}_{\theta}\left[f\left(W_{n+1}\right)\right]=c_{\theta} \sum_{k=1}^{n} \mathbb{E}_{\theta}\left[f\left(W_{n+1}^{k+}\right)-f\left(W_{n+1}^{k-}\right)\right],
$$

where

$$
W_{n+1}^{k \pm}=g_{n}\left(S_{1}, \ldots, S_{k}^{ \pm}, \ldots, S_{n}, A_{2}, \ldots, A_{n+1}\right)
$$

and $S_{k}^{+}$and $S_{k}^{-}$are $S_{k}$-measurable ${ }^{3}$ r.v. distributed according to $\mu_{\theta}^{+}$and $\mu_{\theta}^{-}$, respectively, i.e., $W_{n+1}^{k \pm}$ denotes the $(n+1)^{s t}$ waiting time in a modified queue, where the $k$ th service time $S_{k}$ has been replaced by $S_{k}^{+}$and $S_{k}^{-}$, respectively.

Remark 3 Note that, (14) holds true if $\bar{v}$ is non-decreasing and it satisfies

$$
\forall x, y \geq 0: \bar{v}(x+y) \leq \gamma v(x) v(y)
$$

for some $\gamma>0$. Indeed, note that for all $n \geq 1, g_{n}$ satisfies

$\forall s_{1}, \ldots, s_{n}, t_{1}, \ldots, t_{n} \in \mathbb{R}: g_{n}\left(s_{1}, \ldots, s_{n}, t_{1}, \ldots, t_{n}\right) \leq s_{1}+\cdots+s_{n}+t_{1}+\cdots+t_{n}$

\footnotetext{
${ }^{3}$ I.e., measurable w.r.t. the $\sigma$-field generated by $S_{k}$. In particular, this means that $S_{k}^{ \pm}$are independent of $\left\{S_{i}: i \geq 1, i \neq k\right\}$ and $\left\{T_{j}: j \geq 1\right\}$. However, note that it is not crucial that $S_{k}^{+}$and $S_{k}^{-}$are mutually independent.
} 
Using monotonicity of $\bar{v}$, from (17) we conclude that

$$
\sup _{\substack{s_{1}, \ldots, s_{n} \in \mathbb{R} \\ t_{1}, \ldots, t_{n} \in \mathbb{R}}} \frac{\bar{v}\left(g_{n}\left(s_{1}, \ldots, s_{n}, t_{1}, \ldots, t_{n}\right)\right)}{\prod_{i=1}^{n} v\left(s_{i}\right) \prod_{i=1}^{n} v\left(t_{i}\right)} \leq \gamma^{2 n-1} .
$$

For instance, if for all $x \geq 0 v(x)=\bar{v}(x)=e^{\alpha x}$, for some $\alpha \geq 0$, (17) is fulfilled and we conclude that if $\mu_{\theta}$ is $\mathcal{C}_{v}$-differentiable, then the distribution of $W_{n+1}$ is $\mathcal{C}_{v}$-differentiable, as well.

\section{$1.3\|\cdot\|_{v}$-norm convergence}

Throughout this section we let $v \in \mathcal{C}^{+}$and $\left\{\mu_{\theta}: \theta \in \Theta\right\} \subset \mathcal{M}^{1}$ be such that $v \in \mathcal{L}^{1}\left(\mu_{\theta}: \theta \in \Theta\right)$. The "weak" definition of a limit of measures in (2) has a strong counterpart, where "strong" refers to a norm-setting. More specifically, let $\|\cdot\|$ be a norm on $\mathcal{M}$; we call $\mu_{n}\|\cdot\|$-convergent if $\mu \in \mathcal{M}$ exists such that

$$
\lim _{n \rightarrow \infty}\left\|\mu_{n}-\mu\right\|=0
$$

For example, $\|\mu\|$ may denote the total variation of $\mu$, mentioned in (8). Accordingly, the mapping $\mu_{\theta}$ is called strongly continuous if:

$$
\lim _{\xi \rightarrow 0}\left\|\mu_{\theta+\xi}-\mu_{\theta}\right\|=0
$$

and is called strongly differentiable if there exists $\mu_{\theta}^{\prime} \in \mathcal{M}$, s.t:

$$
\lim _{\xi \rightarrow 0}\left\|\frac{\mu_{\theta+\xi}-\mu_{\theta}}{\xi}-\mu_{\theta}^{\prime}\right\|=0
$$

Note that strong differentiability, as defined in (19), is also referred to as Fréchet differentiability on normed spaces.

For $v \in \mathcal{C}^{+}$, let

$$
\mathbb{S}_{v}:=\{s \in \mathbb{S}: v(s)>0\} .
$$

The $v$-norm of a mapping $g \in \mathcal{C}$ is defined as follows:

$$
\|g\|_{v}:= \begin{cases}\sup _{s \in \mathbb{S}_{v}} \frac{|g(s)|}{v(s)}, & \text { if } g(s)=0 \text { for each } s \notin \mathbb{S}_{v}, \\ \infty, & \text { if there is } s \notin \mathbb{S}_{v} \text { with } g(s) \neq 0 .\end{cases}
$$

It follows that

$$
\|g\|_{v}=\inf \{c>0:|g(s)| \leq c \cdot v(s), \forall s \in \mathbb{S}\}
$$


where we agree that inf $\emptyset=\infty$. Moreover, ${ }^{4}$

$$
\forall s \in \mathbb{S}:|g(s)| \leq\|g\|_{v} \cdot v(s),
$$

and $\mathcal{C}_{v}$ can be described in terms of the $v$-norm, as follows

$$
\mathcal{C}_{v}=\left\{g \in \mathcal{C}:\|g\|_{v}<\infty\right\}
$$

The functional $v$-norm in (20) is extended to measures in the usual way:

$$
\|\mu\|_{v}=\sup _{g \in \mathcal{C}_{v},\|g\|_{v} \leq 1}\left|\int g(s) \mu(d s)\right|=\int v(s)|\mu|(d s),
$$

where $|\mu| \in \mathcal{M}$ is the variation of $\mu$. In the literature it is usually assumed that $\inf \{v(s): s \in \mathbb{S}\}>0$. However, as detailed in the Appendix, we may drop this assumption without any harm. Furthermore, $\mathcal{C}_{v}$ becomes a Banach space when endowed with the $v$-norm. Taking $v \equiv 1$ in (21), i.e., $\mathcal{C}_{v}=\mathcal{C}_{B}$ one recovers the total variation norm $\|\mu\|$ of $\mu$.

Remark 4 The $v$-norm is also known as weighted supremum norm in the literature. An early reference is Lipman (1974). The $v$-norm is frequently used in Markov decision analysis. First traces date back to the early 1980s, see Dekker and Hordijk (1983) and the revised version which was published as Dekker and Hordijk (1988). It was originally used in analysis of Blackwell optimality; see Dekker and Hordijk (1988), and Hordijk and Yushkevich (1999) for a recent publication on this topic. Since then, it has been used in various forms under different names in many subsequent papers, see, for example, Meyn and Tweedie (1993) and Kartashov (1996). For the use of $v$-norm in the theory of measure-valued differentiation of Markov chains, see Heidergott and Hordijk (2003).

$v$-norm-convergence implies $\mathcal{C}_{v}$-weak convergence. For example, it is known that convergence of $\mu_{n}$ towards $\mu$ in total variation norm implies that (2) holds for the set $\mathcal{D}=\mathcal{C}_{B}$. For general $v$ this is a consequence of the Cauchy-Schwartz Inequality. More specifically, for each $\mu \in \mathcal{M}$ s.t. $v \in \mathcal{L}^{1}(\mu)$ it holds that:

$$
\forall g \in \mathcal{C}_{v}:\left|\int g(s) \mu(d s)\right| \leq \int \frac{|g(s)|}{v(s)} \cdot v(s)|\mu|(d s) \leq\|g\|_{v} \cdot\|\mu\|_{v} .
$$

If $\mu_{n}$ converges in $v$-norm to $\mu$, replacing $\mu$ by $\mu_{n}-\mu$ in (22), it follows that (2) holds true for all $g \in \mathcal{C}_{v}$. In words, strong convergence implies weak convergence. The converse is, however, not true; see Heidergott and Leahu (2008) for details.

The following theorem shows that weak differentiability implies strong local Lipschitz continuity.

\footnotetext{
${ }^{4}$ Note that the inequality still holds true if $\|g\|_{v}=\infty$.
} 
Theorem 2 Let $\mu_{\theta}$ be $\mathcal{C}_{v}$-differentiable, with $v \in \mathcal{L}^{1}\left(\mu_{\theta}: \theta \in \Theta\right)$. Then, for any neighborhood $(\theta-\epsilon, \theta+\epsilon) \subset \Theta$ of $\theta$ exists $M_{\epsilon}>0$ such that

$$
\forall|\xi| \leq \epsilon:\left\|\mu_{\theta+\xi}-\mu_{\theta}\right\|_{v} \leq|\xi| M_{\epsilon}
$$

In words, $\mu_{\theta}$ is locally v-norm Lipschitz continuous.

Proof Choose $\epsilon>0$ such that $(\theta-\epsilon, \theta+\epsilon) \subset \Theta$. For $\xi \neq 0$, denote by $T_{\xi}$ the linear continuous functional on $\mathcal{C}_{v}$ defined as:

$$
\forall g \in \mathcal{C}_{v}: T_{\xi} g:=\frac{1}{\xi} \int g(s)\left(\mu_{\theta+\xi}-\mu_{\theta}\right) .
$$

For $\xi \neq 0$ the operator norm of $T_{\xi}$ satisfies:

$$
\left\|T_{\xi}\right\|_{v}=\frac{\left\|\mu_{\theta+\xi}-\mu_{\theta}\right\|_{v}}{|\xi|}
$$

From $\mathcal{C}_{v}$-differentiability of $\mu_{\theta}$ it follows that

$$
\forall g \in \mathcal{C}_{v}: \sup \left\{\left|T_{\xi} g\right|:|\xi| \leq \epsilon\right\}<\infty
$$

and the Banach-Steinhaus Theorem applied to the family $\left\{T_{\xi}:|\xi| \leq \epsilon\right\}$ yields

$$
\sup \left\{\left\|T_{\xi}\right\|_{v}:|\xi| \leq \epsilon\right\}=M_{\epsilon}<\infty
$$

Hence, for $\xi$ such that $|\xi|<\epsilon$ it holds that

$$
\left\|\mu_{\theta+\xi}-\mu_{\theta}\right\|_{v} \leq M_{\epsilon} \cdot|\xi|
$$

which concludes the proof.

As shown by Theorem $2, \mathcal{C}_{v}$-differentiability implies $v$-norm local Lipschitz continuity. This does, however, not imply that $\mathcal{C}_{v}$-differentiability is equivalent to strong $v$-norm differentiability which stems from the fact that

$$
\forall g \in \mathcal{C}_{v}: \lim _{\xi \rightarrow 0} \frac{1}{\xi}\left|\left(\int g(s) \mu_{\theta+\xi}(d s)-\int g(s) \mu_{\theta}(d s)\right)-\int g(s) \mu_{\theta}^{\prime}(d s)\right|=0
$$

does, in general, not imply that

$$
\lim _{\xi \rightarrow 0} \sup _{|g| \leq v}\left|\frac{1}{\xi}\left(\int g(s) \mu_{\theta+\xi}(d s)-\int g(s) \mu_{\theta}(d s)\right)-\int g(s) \mu_{\theta}^{\prime}(d s)\right|=0 .
$$

The following example illustrates this fact. 
Example 5 Consider the uniform distribution $\psi_{\theta}$ on $[0, \theta]$. In Example 3 we have shown that

$$
\psi_{\theta}^{\prime}=\frac{1}{\theta} \delta_{\theta}-\frac{1}{\theta} \psi_{\theta}
$$

Let $v(s)=1$, for $s \in \mathbb{S}=[0, \infty)$, and consider the family of mappings $g_{\xi} \in \mathcal{C}_{v}$ defined as

$$
\forall \xi>0: g_{\xi}(s)= \begin{cases}0, & \text { if } s \leq \theta, \\ \frac{s-\theta}{\xi}, & \text { if } \theta<s \leq \theta+\xi \\ 1, & \text { if } \theta+\xi<s .\end{cases}
$$

It is immediate that for each $\xi>0$ we have $\left\|g_{\xi}\right\|_{v}=1$ and it holds that

$$
\int g_{\xi}(s) \psi_{\theta}(d s)=\int g_{\xi}(s) \psi_{\theta}^{\prime}(d s)=0 .
$$

Therefore,

$$
\left\|\frac{\psi_{\theta+\xi}-\psi_{\theta}}{\xi}-\psi_{\theta}^{\prime}\right\|_{v} \geq\left|\frac{1}{\xi} \int g_{\xi}(s) \psi_{\theta+\xi}(d s)\right|=\frac{1}{2(\theta+\xi)} \rightarrow \frac{1}{2 \theta}>0
$$

Hence, $\mu_{\theta}=\psi_{\theta}$ satisfies (23) but, in general, violates (24) and we conclude that the uniform distribution is an example of a probability measure that is weakly differentiable but fails to be strongly differentiable.

In general, checking strong differentiability, as defined by (19), might be very demanding and it is desirable to have simpler verifiable sufficient conditions for strong differentiability. The following result shows that weak differentiability together with strong continuity of the weak derivative imply strong differentiability. The precise statement is as follows:

Proposition 1 If $\mu_{\theta}$ is weakly $\mathcal{C}_{v}$-differentiable on $\Theta$ such that $\mu_{\theta}^{\prime}$ is v-norm continuous at $\theta$, then $\mu_{\theta}$ is strongly $v$-norm differentiable at $\theta$.

Proof Let $\epsilon>0$ be arbitrary and choose $\varrho>0$ such that

$$
\forall \xi \in(-\varrho, \varrho):\left\|\mu_{\theta+\xi}^{\prime}-\mu_{\theta}^{\prime}\right\|_{v}<\epsilon
$$

Applying the Mean Value Theorem for $\theta \mapsto \int g(s) \mu_{\theta}(d s)$ yields:

$$
\forall \xi \in(-\varrho, \varrho), \forall g \in \mathcal{C}_{v}: \int g(s) \mu_{\theta+\xi}(d s)-\int g(s) \mu_{\theta}(d s)=\xi \int g(s) \mu_{\theta+\xi_{g}}^{\prime},
$$


for some $\xi_{g} \in(-\varrho, \varrho)$ depending on $g$. Hence, for $\|g\|_{v} \leq 1$ it holds that

$$
\begin{aligned}
\mid \int g(s) \mu_{\theta+\xi}(d s) & -\int g(s) \mu_{\theta}(d s)-\xi \int g(s) \mu_{\theta}^{\prime}(d s) \mid \\
& =|\xi| \cdot\left|\int g(s) \mu_{\theta+\xi_{g}}^{\prime}-\int g(s) \mu_{\theta}^{\prime}\right| \leq|\xi| \cdot\left\|\mu_{\theta+\xi_{g}}^{\prime}-\mu_{\theta}^{\prime}\right\|_{v} .
\end{aligned}
$$

Taking in the above inequality the supremmum w.r.t. $g$, we conclude that

$$
\left\|\mu_{\theta+\xi}-\mu_{\theta}-\xi \cdot \mu_{\theta}^{\prime}\right\|_{v} \leq \epsilon|\xi|,
$$

provided that $\xi \in(-\varrho, \varrho)$. Dividing both sides by $|\xi|$ and letting $\xi \rightarrow 0$, proves the claim.

Using Proposition 1, one can easily check, for instance, that for $v(s)=s^{p}$, for some $p \geq 1$, the exponential distribution is strongly differentiable w.r.t. $v$-norm.

\subsection{Bounds on perturbations}

Theorem 2 in Sect. 1.3 establishes strong Lipschitz continuity of weakly differentiable probability measures. For practical purposes one needs the actual Lipschitz constant. The following result shows how a Lipschitz constant can be obtained in explicit form, provided that

$$
\sup _{\theta \in \Theta}\left\|\mu_{\theta}^{\prime}\right\|_{v}<\infty .
$$

Theorem 3 Let $\mu_{\theta}$ be $\mathcal{C}_{v}$-differentiable on $\Theta$ with $v \in \mathcal{L}^{1}\left(\mu_{\theta}: \theta \in \Theta\right)$ and for $\theta_{1}, \theta_{2} \in \Theta$ s.t. $\theta_{1}<\theta_{2}$ set

$$
\mathbf{L}_{\mu}^{v}\left(\theta_{1}, \theta_{2}\right):=\sup _{\theta \in\left[\theta_{1}, \theta_{2}\right]}\left\|\mu_{\theta}^{\prime}\right\|_{v}
$$

(i) Then, for any $g \in \mathcal{C}_{v}$ it holds that

$$
\left|\int g(s) \mu_{\theta_{2}}(d s)-\int g(s) \mu_{\theta_{1}}(d s)\right| \leq \mathbf{L}_{\mu}^{v}\left(\theta_{1}, \theta_{2}\right)\|g\|_{v}\left(\theta_{2}-\theta_{1}\right) .
$$

(ii) In particular, it holds that

$$
\left\|\mu_{\theta_{2}}-\mu_{\theta_{1}}\right\|_{v} \leq \mathbf{L}_{\mu}^{v}\left(\theta_{1}, \theta_{2}\right)\left(\theta_{2}-\theta_{1}\right)
$$

(iii) Let $\mu_{\theta}^{\prime}=\left(c_{\theta}, \mu_{\theta}^{+}, \mu_{\theta}^{-}\right)$. If $g \geq 0$ then one can replace in (26) $\mathbf{L}_{\mu}^{v}\left(\theta_{1}, \theta_{2}\right)$ by

$$
\mathbf{M}_{\mu}^{v}\left(\theta_{1}, \theta_{2}\right):=\sup _{\theta \in\left[\theta_{1}, \theta_{2}\right]}\left(c_{\theta} \cdot \max \left\{\left\|\mu_{\theta}^{+}\right\|_{v},\left\|\mu_{\theta}^{-}\right\|_{v}\right\}\right)
$$


Proof Fix $g \in \mathcal{C}_{v}$. Applying the Mean Value Theorem yields

$$
\left|\int g(s) \mu_{\theta_{2}}(d s)-\int g(s) \mu_{\theta_{1}}(d s)\right|=\left(\theta_{2}-\theta_{1}\right)\left|\int g(s) \mu_{\theta_{g}}^{\prime}(d s)\right|,
$$

for some $\theta_{g} \in\left(\theta_{1}, \theta_{2}\right)$, depending on $g$. On the other hand,

$$
\forall \theta \in\left(\theta_{1}, \theta_{2}\right):\left|\int g(s) \mu_{\theta}^{\prime}(d s)\right| \leq\|g\|_{v} \cdot\left\|\mu_{\theta}^{\prime}\right\|_{v} \leq \mathbf{L}_{\mu}^{v}\left(\theta_{1}, \theta_{2}\right)\|g\|_{v},
$$

according to (22), which concludes (i). Taking in (26) the supremum w.r.t. $\|g\|_{v} \leq 1$, concludes (ii). Finally, for $g \geq 0$ we have

$$
\begin{aligned}
\left|\int g(s) \mu_{\theta}^{\prime}(d s)\right| & =c_{\theta}\left|\int g(s) \mu_{\theta}^{+}(d s)-\int g(s) \mu_{\theta}^{-}(d s)\right| \\
& \leq c_{\theta} \cdot \max \left\{\int g(s) \mu_{\theta}^{+}(d s), \int g(s) \mu_{\theta}^{-}(d s)\right\} \\
& \leq c_{\theta} \cdot \max \left\{\left\|\mu_{\theta}^{+}\right\|_{v},\left\|\mu_{\theta}^{-}\right\|_{v}\right\}\|g\|_{v}
\end{aligned}
$$

which, together with (27), concludes the proof of (iii).

In applications one is often interested in bounds of moments of certain random variables, i.e., one considers $v(s)=s^{p}$, for $s \geq 0$. The following example illustrates how Theorem 3 applies to two usual types of distributions.

Example 6 Let $\mathbb{S}=[0, \infty)$ and $v \in \mathcal{C}(\mathbb{S})$ defined as $v(s)=s^{p}$, for some $p \geq 0$.

(i) Let $\mu_{\theta}$ denote the exponential distribution with rate $\theta$. Recall the definition of $f_{\theta}^{+}$and $f_{\theta}^{-}$given in (9) and (10), respectively. It follows that

$$
\begin{aligned}
\left\|\mu_{\theta}^{\prime}\right\|_{v} & =c_{\theta} \int v(x) f_{\theta}^{+}(x) d x+c_{\theta} \int v(x) f_{\theta}^{-}(x) d x \\
& =\frac{2 e^{-1}+p \Gamma_{\sup }(p+1,1)-p \Gamma_{\inf }(p+1,1)}{\theta^{p+1}},
\end{aligned}
$$

where $\Gamma_{\text {sup }}$ and $\Gamma_{\text {inf }}$ denote the superior (resp., inferior) Gamma functions, which are defined as follows

$$
\forall p>0, x \geq 0: \Gamma_{\text {sup }}(p, x)=\int_{x}^{\infty} s^{p-1} e^{-s} d s, \Gamma_{\mathrm{inf}}(p, x)=\int_{0}^{x} s^{p-1} e^{-s} d s .
$$

Therefore, the Lipschitz constant $\mathbf{L}_{\mu}^{v}\left(\theta_{1}, \theta_{2}\right)$, in Theorem 3 is given by

$$
\mathbf{L}_{\mu}^{v}\left(\theta_{1}, \theta_{2}\right)=\frac{2 e^{-1}+p \Gamma_{\sup }(p+1,1)-p \Gamma_{\inf }(p+1,1)}{\theta_{1}^{p+1}}
$$


and the constant $\mathbf{M}_{\mu}^{v}\left(\theta_{1}, \theta_{2}\right)$ satisfies

$$
\begin{aligned}
\mathbf{M}_{\mu}^{v}\left(\theta_{1}, \theta_{2}\right) & =\sup _{\theta \in\left[\theta_{1}, \theta_{2}\right]} \max \left\{\frac{e^{-1}-p \Gamma_{\mathrm{inf}}(p+1,1)}{\theta^{p+1}}, \frac{e^{-1}+p \Gamma_{\sup }(p+1,1)}{\theta^{p+1}}\right\} \\
& =\frac{e^{-1}+p \Gamma_{\sup }(p+1,1)}{\theta_{1}^{p+1}}
\end{aligned}
$$

In particular, if $p \geq 0$ is an integer it holds that

$$
\mathbf{L}_{\mu}^{v}\left(\theta_{1}, \theta_{2}\right)=\frac{2\left(1+p p ! \sum_{k=0}^{p}(1 / k !)\right)-p p ! e}{\theta_{1}^{p+1} e}
$$

and

$$
\mathbf{M}_{\mu}^{v}\left(\theta_{1}, \theta_{2}\right)=\frac{1+p p ! \sum_{k=0}^{p}(1 / k !)}{\theta_{1}^{p+1} e} .
$$

(ii) For the uniform distribution $\psi_{\theta}$ on $[0, \theta]$, in accordance with Example 3, we obtain the following Lipschitz constants:

$$
\mathbf{L}_{\psi}^{v}\left(\theta_{1}, \theta_{2}\right)=\frac{p+2}{p+1} \theta_{2}^{p-1}
$$

and

$$
\mathbf{M}_{\psi}^{v}\left(\theta_{1}, \theta_{2}\right)=\theta_{2}^{p-1}
$$

Example 6 illustrates the fact that the Lipschitz constants very often depend on the values $\theta_{1}, \theta_{2} \in \Theta$. Thus, from this point of view, notations such as $\mathbf{L}_{\mu}^{v}\left(\theta_{1}, \theta_{2}\right)$ and $\mathbf{M}_{\mu}^{v}\left(\theta_{1}, \theta_{2}\right)$ are justified. However, in what follows we omit specifying the values $\theta_{1}, \theta_{2}$ when not relevant.

We conclude this section by establishing bounds on perturbations for product measures. This is useful when considering performance measures which depend on a finite collection of random variables, as in (1). To this end, let us consider a finite family of measure-valued mappings $\left\{\mu_{i, \theta}: \theta \in \Theta\right\} \subset \mathcal{M}^{1}\left(\mathbb{S}_{i}\right)$, a family of non-negative, continuous mappings $v_{i} \in \mathcal{C}^{+}\left(\mathbb{S}_{i}\right)$ and recall the definitions of $J_{g}(\theta), \Pi_{\theta}$ and $V$ given in (1), (11) and (12), respectively.

Theorem 4 Let $\mu_{i, \theta}$ be weakly $\mathcal{C}_{v_{i}}$-differentiable on $\Theta$, for each $1 \leq i \leq n$ and for arbitrary $\theta_{1}, \theta_{2} \in \Theta$, such that $\theta_{1}<\theta_{2}$ set

$$
\forall 1 \leq i \leq n: \mathbf{L}^{i}=\sup _{\theta \in\left[\theta_{1}, \theta_{2}\right]}\left\|\mu_{\theta, i}^{\prime}\right\|_{v_{i}}
$$


(i) Then, for each $g \in \mathcal{C}_{V}$ it holds that

$$
\left|J_{g}\left(\theta_{2}\right)-J_{g}\left(\theta_{1}\right)\right| \leq \mathbf{L}^{*}\|g\|_{V}\left(\theta_{2}-\theta_{1}\right)
$$

where

$$
\mathbf{L}^{*}=\sum_{i=1}^{n}\left(\mathbf{L}^{i} \prod_{j=1}^{i-1}\left\|\mu_{j, \theta_{2}}\right\|_{v_{j}} \prod_{k=i+1}^{n}\left\|\mu_{k, \theta_{1}}\right\|_{v_{k}}\right)
$$

and we agree that a void product, such as $\prod_{j=1}^{0}\left\|\mu_{j, \theta_{2}}\right\|_{v_{j}}$, equals to 1 .

(ii) In particular, it holds that

$$
\left\|\Pi_{\theta_{2}}-\Pi_{\theta_{1}}\right\|_{V} \leq \mathbf{L}^{*}\left(\theta_{2}-\theta_{1}\right)
$$

(iii) If $g \geq 0$ and for $1 \leq i \leq n, \mu_{i, \theta}^{\prime}=\left(c_{i, \theta}, \mu_{i, \theta}^{+}, \mu_{i, \theta}^{-}\right)$then the constant $\mathbf{L}^{*}$ in (28) can be improved by replacing in (29) $\mathbf{L}^{i}$ by

$$
\mathbf{M}^{i}=\sup _{\theta \in\left[\theta_{1}, \theta_{2}\right]}\left(c_{i, \theta} \cdot \max \left\{\left\|\mu_{i, \theta}^{+}\right\|_{v},\left\|\mu_{i, \theta}^{-}\right\|_{v}\right\}\right) .
$$

Proof For arbitrary $g \in \mathcal{C}_{V}$, we have

$\forall\left(s_{1}, \ldots, s_{n}\right):\left|g\left(s_{1}, \ldots, s_{n}\right)\right| \leq\|g\|_{V} \cdot V\left(s_{1}, \ldots, s_{n}\right)=\|g\|_{V} \cdot v\left(s_{1}\right) \cdot \ldots \cdot v\left(s_{n}\right)$

Therefore, for each $1 \leq i \leq n$, the mapping $g_{i}$ defined as

$$
g_{i}\left(s_{i}\right)=\int \ldots \int g\left(s_{1}, \ldots, s_{n}\right) \prod_{j=1}^{i-1} \mu_{j, \theta_{2}}\left(d s_{j}\right) \prod_{k=i+1}^{n} \mu_{k, \theta_{1}}\left(d s_{k}\right),
$$

belongs to $\mathcal{C}_{v_{i}}$ since, according to Fubini's Theorem, it holds that

$$
\left\|g_{i}\right\|_{v_{i}} \leq\|g\|_{V} \cdot \prod_{j=1}^{i-1}\left\|\mu_{i, \theta_{2}}\right\|_{v_{j}} \cdot \prod_{k=i+1}^{n}\left\|\mu_{k, \theta_{1}}\right\|_{v_{k}}
$$

Since $\mu_{i, \theta}$ is weakly $\mathcal{C}_{v_{i}}$-differentiable, from Theorem 3 (i) we conclude that

$$
\left|\int g_{i}\left(s_{i}\right) \mu_{i, \theta_{2}}\left(d s_{i}\right)-\int g_{i}\left(s_{i}\right) \mu_{i, \theta_{1}}\left(d s_{i}\right)\right| \leq \mathbf{L}^{i}\left\|g_{i}\right\|_{v_{i}}\left(\theta_{2}-\theta_{1}\right) .
$$

On the other hand, simple algebraic calculations show that

$$
J_{g}\left(\theta_{2}\right)-J_{g}\left(\theta_{1}\right)=\sum_{i=1}^{n}\left(\int g_{i}\left(s_{i}\right) \mu_{i, \theta_{2}}\left(d s_{i}\right)-\int g_{i}\left(s_{i}\right) \mu_{i, \theta_{1}}\left(d s_{i}\right)\right) .
$$


Hence, (31) and (30) imply that

$$
\left|J_{g}\left(\theta_{2}\right)-J_{g}\left(\theta_{1}\right)\right| \leq \mathbf{L}^{*}\|g\|_{V}\left(\theta_{2}-\theta_{1}\right)
$$

holds true, for $\mathbf{L}^{*}$ defined by (29), which concludes (i). Finally, a similar reasoning as in Theorem 3 concludes the proof of (ii) and (iii).

Remark 5 If $\mu_{i, \theta}$ is $\mathcal{C}_{v_{i}}$-differentiable, with derivative $\mu_{i, \theta}^{\prime}$, we conclude from Theorem 1 that $J_{g}(\theta)$ is differentiable w.r.t. $\theta$, for each $g \in \mathcal{C}_{V}$. Therefore, one could also derive a Lipschitz bound for $J_{g}(\theta)$ by bounding the derivative $J_{g}^{\prime}(\theta)$ which, according to Theorem 3 can be written as

$$
\sum_{i=1}^{n} \int \ldots \int g\left(s_{1}, \ldots, s_{n}\right) \mu_{i, \theta}^{\prime}\left(d s_{i}\right) \prod_{j \neq i} \mu_{j, \theta}\left(d s_{j}\right)
$$

Using a similar reasoning as in Theorem 4 one would obtain in (28) the following Lipschitz bound:

$$
\mathbf{L}^{\prime}=\sum_{i=1}^{n} \sup _{\theta \in\left[\theta_{1}, \theta_{2}\right]}\left(\left\|\mu_{i, \theta}^{\prime}\right\|_{v_{i}} \prod_{j \neq i}\left\|\mu_{j, \theta}\right\|_{v_{j}}\right)
$$

which, in general, is less accurate (larger) than $\mathbf{L}^{*}$ defined in (29).

Corollary 1 Under the conditions put forward in Theorem 4, if $\mu_{i, \theta}=\mu_{\theta}$ and $v_{i}=v$, for each $1 \leq i \leq n$, then

$$
\mathbf{L}^{*}=\mathbf{L}_{v}^{\mu} \sum_{k=1}^{n}\left\|\mu_{\theta_{2}}\right\|_{v}^{k-1}\left\|\mu_{\theta_{1}}\right\|_{v}^{n-k}, \quad \mathbf{M}^{*}=\mathbf{M}_{v}^{\mu} \sum_{k=1}^{n}\left\|\mu_{\theta_{2}}\right\|_{v}^{k-1}\left\|\mu_{\theta_{1}}\right\|_{v}^{n-k}
$$

\subsection{Application: sensitivity of the average price}

In this section, we consider the following simple example from finance. Assume that an investor is purchasing $S>0$ units worth of stock, each month, for $n \geq 1$ months in a row. If we denote by $X_{k}$ the spot price per share in month $k$, for $1 \leq k \leq n$, then the amount purchased in month $k$ equals to $S / X_{k}$. Hence, the average price per share $X_{a}$, he or she pays over the $n$ months, is obtained by dividing the total amount of wealth spent divided by the total number of shares purchased. That is

$$
X_{a}=\frac{n \cdot S}{\frac{S}{X_{1}}+\cdots+\frac{S}{X_{n}}}=\frac{n}{\frac{1}{X_{1}}+\cdots+\frac{1}{X_{n}}}
$$

i.e., the average price is just the harmonic mean of the spot prices $X_{1}, \ldots, X_{n}$.

Let us fix $n \geq 1$ and assume that $\left\{X_{i}: 1 \leq i \leq n\right\}$ are i.i.d. random variables with distribution $\mu_{\theta}$, depending on some parameter $\theta$. One is interested in studying 
the sensitivity of the expected average price w.r.t. $\theta$, i.e., to obtain a bound for the perturbation

$$
\Delta\left(\theta_{1}, \theta_{2}\right):=\left|\mathbb{E}_{\theta_{2}}\left[X_{a}\right]-\mathbb{E}_{\theta_{1}}\left[X_{a}\right]\right|
$$

for some $\theta_{1}, \theta_{2} \in \Theta$, s.t. $\theta_{1}<\theta_{2}$. To this end, note that the expected average price can be written as

$$
\mathbb{E}_{\theta}\left[X_{a}\right]=J_{h}(\theta)=\int \ldots \int h\left(x_{1}, \ldots, x_{n}\right) \mu_{\theta}\left(d x_{1}\right) \ldots \mu_{\theta}\left(d x_{n}\right)
$$

where $h$ is defined as

$$
\forall x_{1}, \ldots, x_{n}>0: h\left(x_{1}, \ldots, x_{n}\right)=\frac{n}{\frac{1}{x_{1}}+\cdots+\frac{1}{x_{n}}} .
$$

Letting $v \in \mathcal{C}((0, \infty)), v(x)=\sqrt[n]{x}$, it holds that

$$
\forall x_{1}, \ldots, x_{n}>0: h\left(x_{1}, \ldots, x_{n}\right) \leq \sqrt[n]{x_{1} \cdots x_{n}}=V\left(x_{1}, \ldots, x_{n}\right)
$$

where the inequality follows from the well known fact that the harmonic mean of a collection of positive numbers $x_{1}, \ldots, x_{n}$ is smaller than or equal to the geometric mean. Therefore, provided that $\mu_{\theta}$ is weakly $\mathcal{C}_{v}$-differentiable on $\Theta$, Corollary 1 applies and since $h \geq 0$ it holds that; see Theorem 4, (iii)

$$
\begin{array}{r}
\Delta\left(\theta_{1}, \theta_{2}\right) \leq \mathbf{M}^{*}\left(\theta_{2}-\theta_{1}\right) \\
\mathbf{M}^{*}=\mathbf{M}_{v}^{\mu} \sum_{k=1}^{n}\left\|\mu_{\theta_{2}}\right\|_{v}^{k-1}\left\|\mu_{\theta_{1}}\right\|_{v}^{n-k} .
\end{array}
$$

Example 7 If, for instance, $\mu_{\theta}$ is the exponential distribution with rate $\theta$, introduced in Example 2 then we have

$$
\forall \theta \in \Theta:\left\|\mu_{\theta}\right\|_{v}=\frac{1}{\sqrt[n]{\theta}} \Gamma\left(\frac{n+1}{n}\right),
$$

for $v(x)=\sqrt[n]{x}$, where $\Gamma$ denotes the usual Gamma function. Therefore, in accordance with Example 6, we obtain the following Lipschitz constant in (33):

$$
\mathbf{M}^{*}=\frac{\frac{1}{e}+\frac{1}{n} \Gamma_{\sup }\left(\frac{n+1}{n}, 1\right)}{\sqrt[n]{\theta_{1}^{n+1}}} \cdot \frac{\theta_{2}-\theta_{1}}{\sqrt[n]{\theta_{2}}-\sqrt[n]{\theta_{1}}} \cdot\left(\frac{\Gamma\left(\frac{n+1}{n}\right)}{\sqrt[n]{\theta_{1} \theta_{2}}}\right)^{n-1}
$$




\section{Perturbation of waiting times in $G / G / 1$ queue}

Throughout this section we aim to derive bounds on perturbations for the waiting times in the $G / G / 1$ queue. To this end we use the Markov chain description of the sequence of waiting times. A brief overview of Markov operators is provided in Sect. 2.1. In Sect. 2.2, we extend the concept of weak differentiation to Markov operators and we apply the results to derive bounds on perturbations for the transient waiting time in Sect. 2.3.

\subsection{Markov chain description}

Recall that a transition kernel on $\mathbb{S}$ is a mapping $Q: \mathbb{S} \times \mathcal{S} \rightarrow \mathbb{R}$ satisfying:

(i) $\forall E \in \mathcal{S}$, the mapping $Q(\cdot, E)$ is measurable,

(ii) $\forall s \in \mathbb{S}, Q(s, \cdot) \in \mathcal{M}(\mathcal{S})$.

If $Q(s, \cdot) \in \mathcal{M}^{1}$, for all $s \in \mathbb{S}$, we call $Q$ a probability transition kernel (or Markov operator). A transition kernel $Q$ can be identified with a linear operator (denoted also by $Q$ ) on the set of measurable mappings on $\mathbb{S}$, defined as:

$$
\forall s \in \mathbb{S}:(Q f)(s):=\int f(x) Q(s, d x)
$$

for all $f$ for which the right-hand side integral makes sense.

Example 8 We revisit Example 4. From Lindley's recursion we conclude that, for all measurable $f$ it holds that

$$
\mathbb{E}\left[f\left(W_{n+1}\right) \mid W_{n}\right]=\mathbb{E}\left[f\left(\max \left\{W_{n}+S_{n}-A_{n+1}, 0\right\}\right) \mid W_{n}\right]
$$

The Markov operator attached to the sequence of waiting times is thus given by:

$$
\forall x \in \mathbb{S}:(Q f)(x)=\iint f\left((x+s-t)_{+}\right) \eta(d t) \mu(d s),
$$

for suitable $f$ where, we set $y_{+}:=\max \{y, 0\}$, for $y \in \mathbb{R}$.

For $v \in \mathcal{C}^{+}$we introduce the $v$-norm of $Q$ as follows:

$$
\|Q\|_{v}:=\sup _{\|f\|_{v} \leq 1}\|Q f\|_{v}=\sup _{\|f\|_{v} \leq 1} \sup _{s \in \mathbb{S}} \frac{|(Q f)(s)|}{v(s)}
$$

Note that, if $\|Q\|_{v}<\infty$, then it holds that:

$$
\forall f \in \mathcal{C}_{v}:\|Q f\|_{v} \leq\|Q\|_{v} \cdot\|f\|_{v} .
$$


Example 9 Let us consider the Markov operator $Q$ introduced in Example 8 and let $v(x)=e^{\alpha x}$, for some $\alpha \geq 0$. Then

$$
\|Q\|_{v}=\sup _{x \geq 0} e^{-\alpha x} \cdot \mathbb{E}\left[e^{\alpha(x+S-A)_{+}}\right]=\sup _{x \geq 0} \mathbb{E}\left[e^{\alpha\left[(x+S-A)_{+}-x\right]}\right],
$$

where $S$ and $A$ are r.v. distributed according to $\mu$ and $\eta$, respectively. Since the mapping $x \mapsto \alpha\left[(x+S-A)_{+}-x\right]$ is non-increasing, a simple application of the Dominated Convergence Theorem yields:

$$
\|Q\|_{v}=\mathbb{E}\left[e^{e^{\alpha x_{x}\left[(x+S-A)_{+}-x\right]}}\right]=\mathbb{E}\left[e^{\alpha(S-A)_{+}}\right],
$$

provided that the right-hand side expectation above is finite.

We conclude this section by presenting some facts related to composition of kernels. Namely, let $Q_{1}, Q_{2}$ be transition kernels on $\mathbb{S}$ and for $s \in \mathbb{S}$ let:

$$
\left(Q_{2} Q_{1}\right)(s, \cdot):=\int Q_{2}(x, \cdot) Q_{1}(s, d x)
$$

It is straightforward that $Q_{2} Q_{1}$ is a transition kernel on $\mathbb{S}$. Consequently, the induced operator is given by:

$$
\left(Q_{2} Q_{1} f\right)(s)=\int f(y)\left(Q_{2} Q_{1}\right)(s, d y)=\iint f(y) Q_{2}(x, d y) Q_{1}(s, d x) .
$$

One can easily check that $Q_{2} Q_{1} f=Q_{2}\left(Q_{1} f\right)$ and according to (34) we have:

$$
\forall f:\left\|Q_{2} Q_{1} f\right\|_{v} \leq\left\|Q_{2}\right\|_{v} \cdot\left\|Q_{1} f\right\|_{v} \leq\left\|Q_{2}\right\|_{v}\left\|Q_{1}\right\|_{v}\|f\|_{v} .
$$

Taking the supremum w.r.t. $\|f\|_{v} \leq 1$ yields:

$$
\left\|Q_{2} Q_{1}\right\|_{v} \leq\left\|Q_{2}\right\|_{v} \cdot\left\|Q_{1}\right\|_{v} .
$$

In particular, if we set $Q^{n}=\underbrace{Q \ldots Q}_{n}$, for $n \geq 1$, it holds that $\left\|Q^{n}\right\|_{v} \leq\|Q\|_{v}^{n}$.

\subsection{Weak differentiation of Markov operators}

Let $\left\{Q_{\theta}: \theta \in \Theta\right\}$ be a family of Markov operators on $\mathbb{S}$, for some $\Theta \subset \mathbb{R}$. We say that $Q_{\theta}$ is weakly $\mathcal{C}_{v}$-differentiable if there exist a transition kernel $Q_{\theta}^{\prime}$, s.t.

$$
\forall f \in \mathcal{C}_{v}, \forall x \in \mathbb{S}: \frac{d}{d \theta}\left(Q_{\theta} f\right)(x)=\left(Q_{\theta}^{\prime} f\right)(x)
$$


For instance, let $\mathbb{S}=[0, \infty),\left\{\mu_{\theta}: \theta \in \Theta\right\} \subset \mathcal{M}^{1}(\mathcal{S})$ and denote by $Q_{\theta}$ the Markov operator introduced in Example 8, for $\mu=\mu_{\theta}$. That is:

$$
\forall f, \forall x \in \mathbb{S}:\left(Q_{\theta} f\right)(x)=\iint f\left((x+s-t)_{+}\right) \eta(d t) \mu_{\theta}(d s) .
$$

It turns out that weak differentiability of $Q_{\theta}$ is related to that of $\mu_{\theta}$ and one can differentiate $Q_{\theta}$ as follows:

$$
\forall f \in \mathcal{C}_{v}, \forall x \in \mathbb{S}:\left(Q_{\theta}^{\prime} f\right)(x)=\iint f\left((x+s-t)_{+}\right) \eta(d t) \mu_{\theta}^{\prime}(d s) .
$$

This relation will be established by our next result. Specifically, we present a class of mappings $v$ for which $\mathcal{C}_{v}$-differentiability of $\mu_{\theta}$ implies that of $Q_{\theta}$.

Lemma 2 Let $\left\{\mu_{\theta}: \theta \in \Theta\right\} \subset \mathcal{M}^{1}(\mathcal{S}), v \in \mathcal{C}(\mathbb{S})$ be a positive mapping and let $Q_{\theta}$ be defined as in (35). If:

(i) $\mu_{\theta}$ is weakly $\mathcal{C}_{v}$-differentiable,

(ii) for each $x \in \mathbb{S}$ the mapping $v$ satisfies

$$
\sup _{s \in \mathbb{S}} \frac{\int v\left((x+s-t)_{+}\right) \eta(d t)}{v(s)}<\infty,
$$

then $Q_{\theta}$ is weakly $\mathcal{C}_{v}$-differentiable and (36) holds true.

Proof For $s, x \in \mathbb{S}$ and $f \in \mathcal{C}_{v}$ let

$$
H_{f}(s, x)=\int f\left((x+s-t)_{+}\right) \eta(d t)
$$

Condition (ii) essentially says that $\left\|H_{f}(\cdot, x)\right\|_{v}<\infty$ provided that $\|f\|_{v}<\infty$, for all $x \in \mathbb{S}$. To conclude the proof one has to show that $H_{f}(\cdot, x) \in \mathcal{C}_{v}$ for $f \in \mathcal{C}_{v}$, i.e., continuity of $f$ implies continuity of $H_{f}(\cdot, x)$, for each $x \in \mathbb{S}$. To this end, assume that $f$ is continuous, let $s \geq 0$ be fixed and let $\epsilon>0$ be arbitrary. Since continuity of $f$ implies uniform continuity on each compact set (see, e.g., Rudin 1976) it follows that there exists some $\varrho_{\epsilon}>0$ such that for each $s_{1}, s_{2} \in[0, x+s+1]$ it holds that

$$
\left|s_{2}-s_{1}\right|<\varrho_{\epsilon} \Rightarrow\left|f\left(s_{2}\right)-f\left(s_{1}\right)\right|<\epsilon
$$

Therefore, it follows that for any $x \geq 0$ and $|r|<\min \left\{1, \varrho_{\epsilon}\right\}$ we have

$$
\begin{aligned}
\left|H_{f}(s+r, x)-H_{f}(s, x)\right| & =\left|\int f\left((x+s+r-t)_{+}\right)-f\left((x+s-t)_{+}\right) \eta(d t)\right| \\
& \leq \int\left|f\left((x+s+r-t)_{+}\right)-f\left((x+s-t)_{+}\right)\right| \eta(d t) \\
& \leq \int \epsilon \mathbb{I}_{[0, x+s+1]}(t) \eta(d t)=\epsilon \eta([0, x+s+1]),
\end{aligned}
$$


where we used the fact that for $t \geq x+s+1$ and $|r|<1$ we have

$$
(x+s+r-t)_{+}=(x+s-t)_{+}=0 .
$$

Since $\epsilon$ was arbitrary chosen it follows that $H_{f}(\cdot, x)$ is continuous. Hence,

$\forall x \in \mathbb{S}, \forall f \in \mathcal{C}_{v}: \frac{d}{d \theta}\left(Q_{\theta} f\right)(x)=\frac{d}{d \theta} \int H_{f}(s, x) \mu_{\theta}(d s) \stackrel{(i)}{=} \int H_{f}(s, x) \mu_{\theta}^{\prime}(d s)$,

which concludes (36).

Example 10 Let $v(x)=e^{\alpha x}$, for some $\alpha \geq 0$. Since for $s, t, x \geq 0$ it holds that

$$
e^{\alpha(x+s-t)_{+}} \leq e^{\alpha(x+s)},
$$

we obtain

$$
\forall x \geq 0: \sup _{s \in \mathbb{S}} \frac{\int e^{\alpha(x+s-t)+} \eta(d t)}{e^{\alpha s}} \leq e^{\alpha x}<\infty .
$$

Hence, provided that $\mu_{\theta}$ is weakly $\mathcal{C}_{v}$-differentiable, Lemma 2 applies and we conclude that $Q_{\theta}$ is weakly $\mathcal{C}_{v}$-differentiable, as well. Moreover, it holds that:

$$
\begin{aligned}
\left\|Q_{\theta}^{\prime} f\right\|_{v} & =\sup _{x \geq 0} e^{-\alpha x}\left|\iint f\left((x+s-t)_{+}\right) \eta(d t) \mu_{\theta}^{\prime}(d s)\right| \\
& \leq \sup _{x \geq 0} \iint e^{\alpha\left[(x+s-t)_{+}-x\right]}\left|\mu_{\theta}^{\prime}\right|(d s) \eta(d t) \\
& =\iint e^{\alpha(s-t)_{+}}\left|\mu_{\theta}^{\prime}\right|(d s) \eta(d t) \\
& =c_{\theta} \mathbb{E}_{\theta}\left[e^{\alpha\left(S^{+}-A\right)_{+}}+e^{\alpha\left(S^{-}-A\right)_{+}}\right],
\end{aligned}
$$

provided that $\|f\|_{v} \leq 1$, where $\mathbb{E}_{\theta}$ denotes the expectation operator when $S^{+}$and $S^{-}$ are distributed according to $\mu_{\theta}^{+}$and $\mu_{\theta}^{-}$, respectively.

Taking the supremum w.r.t. $\|f\|_{v} \leq 1$, yields

$$
\left\|Q_{\theta}^{\prime}\right\|_{v} \leq c_{\theta} \mathbb{E}_{\theta}\left[e^{\alpha\left(S^{+}-A\right)_{+}}+e^{\alpha\left(S^{-}-A\right)_{+}}\right]
$$

\subsection{The transient waiting time}

In what follows we apply the results from previous section in order to derive bounds on perturbations for the expected waiting times in a $G / G / 1$ queue.

Let us consider the $G / G / 1$ queue, as introduced in Example 8 and let us assume that the service time distribution $\mu_{\theta}$ depends on some design parameter $\theta \in \Theta$. Assuming 
that $\theta_{1}<\theta_{2}$, we aim to establish bounds for

$$
\forall x, f, n: \Delta_{n}^{x, f}\left(\theta_{1}, \theta_{2}\right):=\left|\mathbb{E}_{\theta_{2}}\left[f\left(W_{n+1}^{x}\right)\right]-\mathbb{E}_{\theta_{1}}\left[f\left(W_{n+1}^{x}\right)\right]\right|,
$$

for arbitrary $n \geq 1, x \geq 0$ and $f \in \mathcal{C}_{v}$, where $W_{n}^{x}$ denotes the $n$th waiting time, when $W_{1}^{x}=x$ and $\mathbb{E}_{\theta}$ denotes the expectation operator, when the service times follow distribution $\mu_{\theta}$. To do so, let us consider the Markov operator $Q_{\theta}$ as introduced in (35) and note that, for any initial state $x \geq 0$, it holds that:

$$
\forall n \geq 1, \forall g: \mathbb{E}_{\theta}\left[g\left(W_{n+1}^{x}\right)\right]=\mathbb{E}\left[\mathbb{E}_{\theta}\left[g\left(W_{n+1}^{x}\right) \mid W_{1}^{x}\right]\right]=\left(Q_{\theta}^{n} g\right)(x)
$$

Hence, the expression in (38) can be re-written and bounded as follows:

$$
\left|\left(Q_{\theta_{2}}^{n} f\right)(x)-\left(Q_{\theta_{1}}^{n} f\right)(x)\right| \leq\left\|Q_{\theta_{2}}^{n}-Q_{\theta_{1}}^{n}\right\|_{v} \cdot\|f\|_{v} \cdot v(x) .
$$

Elementary algebraic calculations show that:

$$
Q_{\theta_{2}}^{n}-Q_{\theta_{1}}^{n}=\sum_{k=1}^{n} Q_{\theta_{2}}^{n-k}\left(Q_{\theta_{2}}-Q_{\theta_{1}}\right) Q_{\theta_{1}}^{k-1}
$$

where we agree that $Q^{0}$ is the identity operator ${ }^{5}$. Hence:

$$
\left\|Q_{\theta_{2}}^{n}-Q_{\theta_{1}}^{n}\right\|_{v} \leq \sum_{k=1}^{n}\left\|Q_{\theta_{2}}\right\|_{v}^{n-k}\left\|Q_{\theta_{2}}-Q_{\theta_{1}}\right\|_{v}\left\|Q_{\theta_{1}}\right\|_{v}^{k-1}
$$

Provided that $Q_{\theta}$ is $\mathcal{C}_{v}$-differentiable on $\Theta$, one can apply the Mean Value Theorem to the mapping $\theta \mapsto\left(Q_{\theta} g\right)(x)$, which yields

$$
\forall x \in \mathbb{S}, g \in \mathcal{C}_{v}:\left(Q_{\theta_{2}} f\right)(x)-\left(Q_{\theta_{1}} f\right)(x)=\left(\theta_{2}-\theta_{1}\right) \cdot\left(Q_{\theta}^{\prime} g\right)(x),
$$

for some $\theta \in\left(\theta_{1}, \theta_{2}\right)$, depending on $g$ and $x$. Thus, for $\|g\|_{v} \leq 1$ we have:

$$
\left\|\left(Q_{\theta_{2}} g\right)-\left(Q_{\theta_{1}} g\right)\right\|_{v} \leq\left(\theta_{2}-\theta_{1}\right)\left\|Q_{\theta}^{\prime}\right\|_{v} \leq\left(\theta_{2}-\theta_{1}\right) \sup _{\theta \in\left[\theta_{1}, \theta_{2}\right]}\left\|Q_{\theta}^{\prime}\right\|_{v} .
$$

Consequently, taking the supremum w.r.t. $\|g\|_{v} \leq 1$ yields:

$$
\left\|Q_{\theta_{2}}-Q_{\theta_{1}}\right\|_{v} \leq\left(\theta_{2}-\theta_{1}\right) \sup _{\theta \in\left[\theta_{1}, \theta_{2}\right]}\left\|Q_{\theta}^{\prime}\right\|_{v} .
$$

Therefore, from (39) together with (40) and (41) we conclude that

$$
\left|\left(Q_{\theta_{2}}^{n} f\right)(x)-\left(Q_{\theta_{1}}^{n} f\right)(x)\right| \leq \mathbf{C}_{n} \mathbf{L}\|f\|_{v}\left(\theta_{2}-\theta_{1}\right) v(x),
$$

\footnotetext{
5 The identity operator corresponds to 'Dirac' transition kernel $\mathbf{1}(x, A)=\delta_{x}(A)$. In particular, $\mathbf{1} f=f$, for all measurable $f$.
} 
where

$$
\mathbf{C}_{n}=\sum_{k=1}^{n}\left\|Q_{\theta_{2}}\right\|_{v}^{n-k}\left\|Q_{\theta_{1}}\right\|_{v}^{k-1}, \quad \mathbf{L}=\sup _{\theta \in\left[\theta_{1}, \theta_{2}\right]}\left\|Q_{\theta}^{\prime}\right\|_{v}
$$

Finally, we obtain the following bound for the expression in (38):

$$
\Delta_{n}^{x, f}\left(\theta_{1}, \theta_{2}\right) \leq \mathbf{C}_{n} \mathbf{L}\|f\|_{v}\left(\theta_{2}-\theta_{1}\right) v(x) .
$$

Example 11 Let us consider a $M / U / 1$ queue where service times have uniform distribution $\psi_{\theta}$, on $[0, \theta]$ and inter-arrival times are exponentially distributed with rate $\lambda$, i.e., the Markov operator $Q_{\theta}$ is given by

$$
\forall f, \forall x \in \mathbb{S}:\left(Q_{\theta} f\right)(x)=\frac{1}{\theta} \int_{0}^{\theta} \int_{0}^{\infty} f\left((x+s-t)_{+}\right) \lambda e^{-\lambda t} d t d s .
$$

Then, according to Example 9, for $v(x)=e^{\alpha x}$, for some $\alpha \geq 0$, it holds that

$$
\forall \lambda, \theta, \alpha:\left\|Q_{\theta}\right\|_{v}=\mathbb{E}_{\theta}\left[e^{\alpha(S-A)_{+}}\right]=\frac{\lambda^{2}\left[e^{\alpha \theta}-1\right]+\alpha^{2}\left[1-e^{-\lambda \theta}\right]}{\alpha \lambda(\alpha+\lambda) \theta} .
$$

Similarly, according to (37) we conclude that

$$
\forall \lambda, \theta, \alpha:\left\|Q_{\theta}^{\prime}\right\|_{v} \leq \frac{\lambda^{2}\left[(1+\alpha \theta) e^{\alpha \theta}-1\right]+\alpha^{2}\left[1-(1+\lambda \theta) e^{-\lambda \theta}\right]}{\alpha \lambda(\alpha+\lambda) \theta}
$$

and a bound for the perturbation in (38) can be obtained according to (43).

To illustrate the above findings we let $\alpha=\lambda$ and we obtain

$$
\forall \lambda, \theta:\left\|Q_{\theta}\right\|_{v}=\frac{1}{\lambda \theta} \sinh (\lambda \theta),\left\|Q_{\theta}^{\prime}\right\|_{v} \leq \frac{1+\lambda \theta}{\lambda \theta} \sinh (\lambda \theta)
$$

where sinh denotes the hyperbolic sine function. Consequently, we have

$$
\mathbf{C}_{n}=\sum_{k=1}^{n}\left(\frac{\sinh \left(\lambda \theta_{2}\right)}{\lambda \theta_{2}}\right)^{n-k}\left(\frac{\sinh \left(\lambda \theta_{1}\right)}{\lambda \theta_{1}}\right)^{k-1}, \quad \mathbf{L} \leq \frac{1+\lambda \theta_{2}}{\lambda \theta_{2}} \sinh \left(\lambda \theta_{2}\right)
$$

where we use the fact that the function $x \mapsto \frac{1+x}{x} \sinh (x)$ is non-decreasing in $x$. Substituting the above constants in (43) yields

$$
\frac{\Delta_{n}^{x, f}\left(\theta_{1}, \theta_{2}\right)}{\left(\theta_{2}-\theta_{1}\right)} \leq\|f\|_{v}\left(1+\lambda \theta_{2}\right) e^{\lambda x} \sum_{k=1}^{n}\left(\frac{\sinh \left(\lambda \theta_{2}\right)}{\lambda \theta_{2}}\right)^{k}\left(\frac{\sinh \left(\lambda \theta_{1}\right)}{\lambda \theta_{1}}\right)^{n-k} .
$$




\section{Summary}

We studied bounds on cost functions over finite products of probability measures. The main contribution of our analysis are the following. We show that the classical $v$-norm can be extended to mappings $v$ such that $\inf _{s \in \mathbb{S}} v(s)=0$. We provide a through study of the relation between weak differentiability and Fréchet differentiability with respect to the $v$-norm. In particular, by applying a fundamental result from the theory of Banach spaces, we show that weak differentiability implies $v$-norm Lipschitz continuity. This result leads to bounds on the sensitivity of finite products of probability measures in norm sense. We illustrate the application of these results by providing bounds for perturbations of the $n$th waiting time in an $\mathrm{G} / \mathrm{G} / 1$ queue. The extension of our bound to the stationary waiting time is topic of further research.

Open Access This article is distributed under the terms of the Creative Commons Attribution Noncommercial License which permits any noncommercial use, distribution, and reproduction in any medium, provided the original author(s) and source are credited.

\section{Appendix}

\section{Uniqueness of the $\mathcal{C}_{v}$-limit}

The $\mathcal{C}_{v}$-limit in (2) is in general not unique. Indeed, let us consider $\mathbb{S}=[0, \infty)$ endowed with the usual metric, and $v(s)=s$, for all $s \in \mathbb{S}$, and denote by $\delta_{0}$ the Dirac measure, i.e., $\delta_{0}$ assigns mass 1 to point 0 . Assume that $\mu$ is a $\mathcal{C}_{v}$-limit of the sequence $\left\{\mu_{n}\right\}_{n} \subset \mathcal{M}$. Since for $g \in \mathcal{C}_{v}$ we have $g(0)=0, \mu+\alpha \cdot \delta_{0} \in \mathcal{M}$ is also a $\mathcal{C}_{v}$-limit of the sequence $\left\{\mu_{n}\right\}_{n}$, for each $\alpha \in \mathbb{R}$ and the $\mathcal{C}_{v}$-limit fails to be unique. In words, (2) still holds true if one assigns a different mass on the 'zero set' of $v$. Our next result will elucidate this uniqueness issue. In particular it shows that the set $\mathcal{C}_{v}$, likewise $\mathcal{C}_{B}$, is appropriate for introducing weak convergence.

Lemma 3 Let $v \in \mathcal{C}$ and $v \geq 0$. Then the set $\mathcal{C}_{v}$ is a Banach space, when endowed with the v-norm and the $\mathcal{C}_{v}$-limit is uniquely determined on $\mathbb{S}_{v}$.

Proof Let $\Phi: \mathcal{C}_{v} \rightarrow \mathcal{C}_{B}$ be defined by

$$
(\Phi g)(s)= \begin{cases}\frac{g(s)}{v(s)} & s \in \mathbb{S}_{v} \\ \lim _{t \rightarrow s} \frac{g(t)}{v(t)} & \text { otherwise }\end{cases}
$$

Then, $\Phi$ is an isometry ${ }^{6}$, i.e., a bijection such that

$$
\|\Phi g\|_{\infty}=\|g\|_{v}
$$

Since, $\mathcal{C}_{B}$ equipped with the $\|\cdot\|_{\infty}$-norm is a Banach space, it follows that $\mathcal{C}_{v}$ equipped with the $\|\cdot\|_{v}$-norm is a Banach space as well; For details, see Semadeni (1971).

\footnotetext{
6 The condition $v \in \mathcal{C}$ ensures that $\Phi g \in \mathcal{C}$, for $g \in \mathcal{C}$.
} 
Let now $\left\{\mu_{n}\right\}_{n} \subset \mathcal{M}$ be such that $v \in \mathcal{L}^{1}\left(\mu_{n}: n \in \mathbb{N}\right)$ and $\mu, \eta \in \mathcal{M}$ be $\mathcal{C}_{v}$-limits of the sequence $\left\{\mu_{n}\right\}_{n}$. We will show that:

$$
\forall E \in \mathcal{S}: E \subset \mathbb{S}_{v} \Rightarrow \mu(E)=\eta(E)
$$

First, since $\mu$ and $\eta$ are $\mathcal{C}_{v}$-limits of the same sequence, (2) yields:

$$
\forall g \in \mathcal{C}_{v}: \int g(s) \mu(d s)=\int g(s) \eta(d s)
$$

Next, for arbitrary $E \in \mathcal{S}$ let $\mathbb{I}_{E}$ denote its indicator function and set, for $\gamma>0$ :

$$
h_{\gamma}^{E}(s)=\min \left\{\gamma \cdot v(s), \mathbb{I}_{E}(s)\right\}, \forall s \in \mathbb{S} .
$$

The fact that $\left|h_{\gamma}^{E}(s)\right| \leq \gamma \cdot v(s)$, for $s \in \mathbb{S}$, together with $E \subset \mathbb{S}_{v}$ implies $h_{\gamma}^{E} \in \mathcal{C}_{v}$. For $v \in \mathcal{L}^{1}(\mu, \eta)$ it follows that $h_{\gamma}^{E} \in \mathcal{L}^{1}(\mu, \eta)$, for all $\gamma>0$. Moreover, it holds that:

$$
\forall s \in \mathbb{S}, \forall E \in \mathcal{S}: \lim _{\gamma \rightarrow \infty} h_{\gamma}^{E}(s)= \begin{cases}\mathbb{I}_{E}(s) & s \in \mathbb{S}_{v} \\ 0 & \text { otherwise }\end{cases}
$$

Hence $h_{\gamma}^{E} \uparrow \mathbb{I}_{E \cap \mathbb{S}_{v}}$, for $\gamma \uparrow \infty$, and the Monotone Convergence Theorem together with (45) yields:

$$
\forall E \subset \mathbb{S}_{v}: \mu(E)=\lim _{\gamma \rightarrow \infty} \int h_{\gamma}^{E}(s) \mu(d s)=\lim _{\gamma \rightarrow \infty} \int h_{\gamma}^{E}(s) \eta(d s)=\eta(E),
$$

which concludes (44).

The above result can be phrased as follows: the trace of the $\mathcal{C}_{v}$-limit $\mu$ is unique on the set $\mathbb{S}_{v}$. More formally, if we denote by $\equiv$ the equivalence relation on $\mathcal{M}$ given by

$$
\mu \equiv \eta \quad \text { iff } \forall E \subset \mathbb{S}_{v}: \mu(E)=\eta(E)
$$

then the $\mathcal{C}_{v}$-limit is uniquely determined up to this equivalence relation and the precise definition of the $\mathcal{C}_{v}$-limit would be in terms of the equivalence class of $\mu$, denoted by $[\mu]$. Note that since $g \in \mathcal{C}_{v}$ implies $g(s)=0$ for $s \notin \mathbb{S}_{v}$, the behavior of $\mu$ outside $\mathbb{S}_{v}$ is not relevant for our analysis and we may, with slight abuse of notation, identify $\mu$ and $[\mu]$. Lemma 3 shows that:

$$
\left[\mu_{n}\right](\mathbb{S})=0, \forall n \in \mathbb{N} \Rightarrow[\mu](\mathbb{S})=0 .
$$

In fact, the algebraic dual space $\mathcal{C}_{v}^{*}$ of $\mathcal{C}_{v}$ (i.e., the set of all linear functionals on $\mathcal{C}_{v}$ ) is the quotient space of $\mathcal{M}$ with respect to equivalence relation $\equiv$. 


\section{References}

Aissani D, Bouallouche-Medjkoune L (2006) Performance analysis approximation in a queueing system of type M/G/1. Math Methods Oper Res 63(2):341-356

Billingsley B (1966) Weak convergence of probability measures. Wiley, New York

Cho GE, Meyer CD (2001) Comparison of perturbation bounds for the stationary distribution of a Markov chain. Linear Algebra Appl 335:137-150

Dekker R, Hordijk A (1983) Average, sensitive and Blackwell optimal policies in denumerable Markov decision chains with unbounded rewards. Report no.83-36, Institute of Applied Mathematics and Computing Science

Dekker R, Hordijk A (1988) Average, sensitive and Blackwell optimal policies in denumerable Markov decision chains with unbounded rewards. Math Oper Res 13:395-421

Heidergott B, Hordijk A (2003) Taylor series expansions for stationary Markov chains. Adv Appl Probab 23:1046-1070

Heidergott B, Hordijk A (2004) Single-run gradient estimation via measure-valued differentiation. IEEE Trans Automat Control 49:1843-1846

Heidergott B, Leahu H (2008) Differentiability of product measures. FEWEB Research Memorandum 2008-5, Vrije Universiteit Amsterdam, The Netherlands

Hordijk A, Yushkevich AA (1999) Blackwell optimality in the class of all policies in Markov decision chains with a Borel state space and unbounded rewards. Math Oper Res 50:421-448

Kartashov N (1996) Strong stable Markov chains. VSP, Utrecht

Kushner H, Vázquez-Abad F (1992) Estimation of the derivative of a stationary measure with respect to a control parameter. J Appl Probab 29:343-352

Lipman S (1974) On dynamic programming with unbounded rewards. Mange Sci 21:1225-1233

Meyn SP, Tweedie RL (1993) Markov chains and stochastic stability. Springer, London

Pflug G (1996) Optimization of stochastic models. Kluwer, Boston

Rudin W (1976) Principles of mathematical analysis, 3rd edn. McGraw-Hill, Tokyo

Schweitzer PJ (1968) Perturbation theory and finite Markov chains. J Appl Prob 5:401-413

Semadeni Z (1971) Banach spaces of continuous functions. Polish scientific publishers, Warszawa 